\title{
BMJ Open Adjunctive inspiratory muscle training for patients with COVID-19 (COVIDIMT): protocol for randomised controlled double-blind trial
}

\author{
Vinicius Maldaner (D) , ${ }^{1,2}$ Jean Coutinho, ${ }^{2}$ Alfredo Nicodemos da Cruz Santana, ${ }^{3}$ \\ Graziella F B Cipriano, ${ }^{4}$ Miriã Cândida Oliveira (1) , ${ }^{2}$ Marilúcia de Morais Carrijo, ${ }^{2}$ \\ Maria Eduarda M Lino, ${ }^{2}$ Lawrence P Cahalin, ${ }^{5}$ Alexandra GCB Lima, ${ }^{6}$ \\ Robson Borges, ${ }^{4}$ Dante Brasil Santos, ${ }^{2}$ Iransé Oliveira Silva, ${ }^{2,7}$ \\ Luis Vicente Franco Oliveira, ${ }^{1,2}$ Gerson Cipriano Jr. ${ }^{4,8}$
}

To cite: Maldaner V, Coutinho J, Santana ANdC, et al. Adjunctive inspiratory muscle training for patients with COVID-19 (COVIDIMT): protocol for randomised controlled double-blind trial. BMJ Open 2021;11:e049545. doi:10.1136/ bmjopen-2021-049545

- Prepublication history for this paper is available online. To view these files, please visit the journal online (http://dx.doi. org/10.1136/bmjopen-2021049545).

Received 30 January 2021 Accepted 15 June 2021

Check for updates

(C) Author(s) (or their employer(s)) 2021. Re-use permitted under CC BY-NC. No commercial re-use. See rights and permissions. Published by BMJ.

For numbered affiliations see end of article.

Correspondence to Dr Vinicius Maldaner; viniciusmaldaner@gmail.com

\section{ABSTRACT}

Introduction A significant number of patients with COVID-19 may experience dyspnoea, anxiety, depression, pain, fatigue and physical impairment symptoms, raising the need for a multidisciplinary rehabilitation approach, especially for those with advanced age, obesity, comorbidities and organ failure. Traditional pulmonary rehabilitation (PR), including exercise training, psychosocial counselling and education, has been employed to improve pulmonary function, exercise capacity and quality of life in patients with COVID-19. However, the effects of inspiratory muscle training (IMT) in PR programmes remain unclear. This study aimed to determine whether the addition of a supervised IMT in a PR is more effective than PR itself in improving dyspnoea, health-related quality of life and exercise capacity in symptomatic patients with postCOVID-19.

Methods and analysis This parallel-group, assessorblinded randomised controlled trial, powered for superiority, aimed to assess exercise capacity as the primary outcome. A total of 138 are being recruited at two PR centres in Brazil. Following baseline testing, participants will be randomised using concealed allocation, to receive either (1) standard PR with sham IMT or (2) standard PR added to IMT. Treatment effects or differences between the outcomes (at baseline, after 8 and 16 weeks, and after 6 months) of the study groups will be analysed using an ordinary two-way analysis of variance.

Ethics and dissemination This trial was approved by the Brazilian National Ethics Committee and obtained approval on 7 0ctober 2020 (document number 4324069). The findings will be disseminated through publications in peerreviewed journals and conference presentations. Trial registration number NCT04595097.

\section{INTRODUCTION}

COVID-19 has rapidly evolved into a global health emergency. The disease progression varies widely, with the patients exhibiting different symptoms such as hypoxia, dyspnoea and fatigue. ${ }^{1}$ A significant number of patients

\section{Strengths and limitations of this study}

Evaluation of economic efficiency in addition to clinical effectiveness.

- The first fully powered study comparing inspiratory muscle training (IMT) combined with pulmonary rehabilitation (PR) with sham IMT combined with PR in patients with post-COVID 19.

Full therapist blinding will be not possible.

with COVID-19 will have dyspnoea, anxiety, depression, pain, fatigue and physical impairment symptoms, which may be related to postintensive care syndrome (PICS). ${ }^{2}$ The aforementioned symptoms and limitations of the PICS may persist for years, like in patients without COVID-19 with acute respiratory distress syndrome. Persistent and late-onset symptoms in patients with post-COVID-19 may persist for more than 120 days after symptom onset. ${ }^{3}$ Recent literature examining the consequences of infectious diseases like severe acute respiratory syndrome and COVID-19 has identified respiratory muscle weakness and low exercise capacity that persists for a long time after the initial infection as residual effects. ${ }^{4}$

Further research is needed to identify whether treatment strategies can reduce the duration of symptoms after COVID-19. Recent SARS-CoV-2 experience has revealed the need for a multidisciplinary rehabilitation approach, especially for patients with advanced age, obesity, comorbidities and organ failure. ${ }^{5}$

The International European Respiratory Society (ERS) and the American Thoracic Society (ATS) Task Force suggest that 
COVID-19 survivors with a need for rehabilitative interventions at $6-8$ weeks following hospital discharge should receive a comprehensive pulmonary rehabilitation (PR) programme. ${ }^{6}$ Although most studies have evaluated the effects of PR in patients with chronic pulmonary obstructive disease (COPD), evidence suggests that it is a treatment option for other pulmonary conditions such as idiopathic pulmonary fibrosis (IPF) and COVID-19. ${ }^{78}$ PR has been advocated to provide comprehensive care, improve functional status, reduce symptoms, and decrease healthcare usage in patients with respiratory diseases. PR involves exercise training, psychosocial counselling and education, and recently, the ERS advocated that inspiratory muscle training (IMT) should be considered as an additional intervention in PR programmes, especially in patients with inspiratory muscle weakness. ${ }^{9}$

As dyspnoea and fatigue are two of the most common late-onset symptoms of COVID-19, strategies to treat these symptoms should be encouraged. Langer et al showed that 8 weeks of partially supervised IMT improved respiratory muscle strength and endurance, dyspnoea, and exercise endurance in subjects with COPD. ${ }^{10} \mathrm{~A}$ recent meta-analysis also demonstrated positive results for dyspnoea and Health-related quality of life (HRQoL) in 362 patients with interstitial pulmonary fibrosis, associated with IMT with aerobic exercise. ${ }^{8}$ A recent review discussed the use of IMT in symptomatic patients with respiratory muscle weakness who are motivated to optimise their functional capacity gains through a PR programme. Thus, randomised controlled trials (RCTs) should be conducted to examine the addition of IMT in PR programmes in patients with exercise-induced dyspnoea and low functional capacity.

Notably, few controlled clinical trials have investigated the addition of IMT in PR for patients after viral pneumonia illnesses, including COVID-19. In view of this fact, this trial aims to determine if supervised IMT added to PR is more effective than PR alone in improving dyspnoea, HRQoL and exercise capacity in symptomatic patients with post-COVID-19. We hypothesise that the addition of IMT in PR programmes will improve respiratory muscle strength, peak $\mathrm{VO}_{2}$, breathlessness and be cost-effective compared with traditional PR. This protocol is reported according to the Standard Protocol Items: Recommendations for Interventional Trials (SPIRIT guidance).

\section{METHODS AND ANALYSIS}

\section{Study design}

This study consists of a pragmatic randomised, doubleblind controlled trial with two parallel groups (arms), conducted at three PR centres in the cities of Anapolis and Brasilia, Brazil. This protocol follows SPIRIT guidelines. A flowchart of the study protocol is shown in figure 1 .

\section{Patient and public involvement}

Patients and the public will not be directly involved in the design, recruitment or conduct of this study. They will be

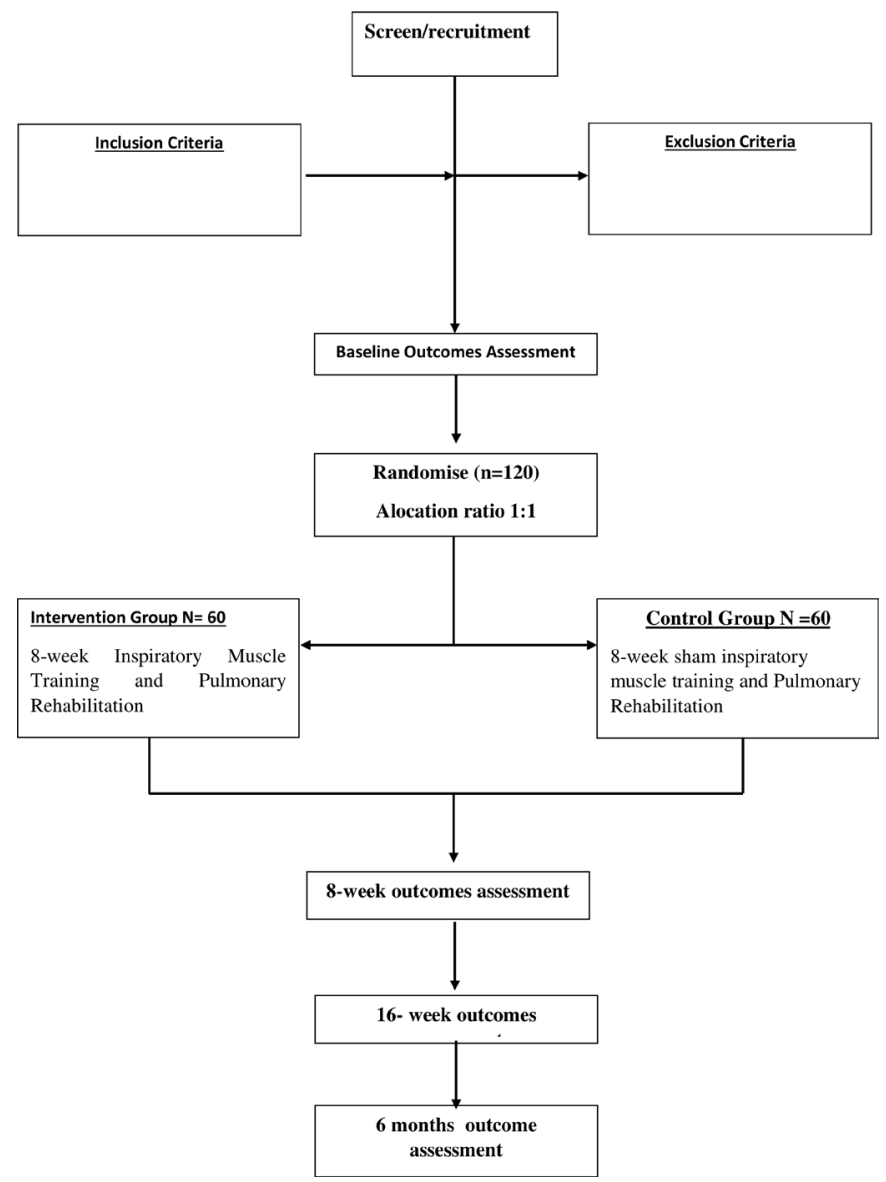

Figure 1 Flowchart.

involved in our plans to disseminate the study results to participants and relevant community groups by assisting in the choice of what information/results to share and in what format.

\section{Participants}

Eligible participants will be identified through screening 4-6 weeks after ICU discharge. They will be screened by pulmonologists of long-term COVID-19 ambulatory in hospitals located in Brasilia and Anapolis. To be eligible, participants must be over 18 years of age with a confirmed COVID-19 diagnosis that required hospitalisation and either (1) non-invasive respiratory support (Continous Positive AirWay Pressure (CPAP), high-flow oxygen catheter, non-breathing oxygen mask or (2) invasive mechanical ventilation within 3 months of study recruitment. The exclusion criteria will include pregnancy, dependence on others to perform activities of daily living during the month prior to the latest intensive care unit (ICU) admission (gait aids are acceptable); documented cognitive impairment; proven or suspected spinal cord injury or other neuromuscular diseases that will result in a permanent or prolonged weakness (not including ICU-acquired weakness); severe neurological disease; death being deemed inevitable as a result of the current illness; and unwillingness to commit to full active treatment either on the part of the patient or the treatment decision-maker. 
For enrolment into the study, informed consent will be sought from the patients according to Brazil's federal laws.

\section{Randomisation and allocation}

Following informed consent and all baseline assessments, participants will be randomised at a 1:1 rate, according to the block randomisation generated by the website (https://www.sealedenvelope.com) to either the first intervention group (PR combined with IMT) or the control group (PR combined with sham IMT). An independent researcher of the trial will prepare the randomisation schedule. The study registration was in March 2021; the final registration will be in May 2022.

\section{Ethics and dissemination}

The project was approved by the Brazilian National Ethics Committee and received approval on 7 October 2020 (document number 4324069). This trial was prospectively registered in the Clinical Trials Registry Platform on 20 October 2020 (NCT04595097); updated on 12 April 2021. Patients will be invited to participate voluntarily. The results of this study will be reported in full through peer-reviewed journals and presented at scientific conferences. Significant protocol modifications will be communicated to the participants, trial registers and journals. A copy of the informed consent form will be provided, if requested.

\section{Consent for publication and confidentiality}

All information collected from the study participants will be kept confidential and stored in the laboratory's database during and after the trial. Only the researchers will assess the data to ensure anonymity and respect for human dignity and fulfil all the bioethics requirements of Resolution 466/2012 of the National Health Council and the Helsinki Declaration for research with humans.

\section{Blinding}

A trained physical therapist, blinded to the intervention allocation group, will perform all outcome assessments. One arm of the study will undergo a sham treatment (described further), which characterises the double-blind trial.

\section{Interventions}

The intervention consists of a supervised intervention with a workload of $50 \%-60 \%$ of the maximal inspiratory pressure (MIP), including two sets of 30 breaths ( 60 breaths daily), with a 2 min interval in between work sets, 3 days/week, using a tapered flow-resistive loading device (POWERbreathe KH2, HaB International, UK). This device was chosen mainly considering its visual feedback capability and the possibility of controlling the key training parameters (eg, number of repetitions, external resistance, volume response and work performed) during and after training sessions. It allows frequent reassessment of the patient's training parameters for adjusting the training. The IMT programme will be associated with a PR programme, following a previously published protocol, for 8 weeks.

Patients will be instructed and encouraged to perform 30 fast and effective inspirations and will be allowed to pause if necessary, though not exceeding $1 \mathrm{~min}$. The MIP and maximal dynamic inspiratory pressure (S-Index) will be updated weekly to maintain the training load at the highest tolerable load, $50 \%-60 \%$ of the most recent MIP. Individuals will perform weekly MIP assessments to ensure the correct load training. The same trained physiotherapist will perform all the respiratory muscle strength assessments. The training workload of the control group will be set at $10 \%$ of the baseline MIP and will not be modified throughout the intervention period. ${ }^{11}$ The IMT protocol will be performed after PR.

Both groups will perform the same PR programme. The PR staff will prescribe a tailored individualised PR programme within the prespecified parameters recommended by the PR guidelines. The PR will be delivered three times a week, for 8 weeks (maximum 24 sessions (26 sessions total, including familiarisation sessions)), of 30-50 min supervised exercise sessions, with progressive and individualised multimodal exercises (including both aerobic, strength training and functional fitness modalities). An adequate $5 \mathrm{~min}$ warm-up and $5 \mathrm{~min}$ cool-down will be incorporated. According to previously determined ventilatory thresholds during a maximal cardiopulmonary exercise test, the intensity will be individually adjusted in the moderate domain and monitored by heart rate (HR) monitoring (10\% range of the HR at first ventilatory threshold, rating of perceived exertion (4-6 in Borg scale) and oxygen saturation (above $90 \%$ ), respectively.

Experienced physical therapists will perform familiarisation sessions to optimise exercise prescription. The adherence to the PR programme will be recorded in a daily $\log$.

\section{Measures}

The initial assessment will be performed at the pulmonology department at hospitals in Brasilia and Anapolis. Patients will receive a routine check-up, and if necessary, current medications will be optimised. Any changes to medication will be documented. Adverse events and complications during rehabilitation will be recorded by the physicians on a standardised basis in the medical survey sheet at the end of rehabilitation. Anthropometric and sociodemographic data will be collected in the first assessment.

\section{Primary outcome measures}

Cardiopulmonary exercise testing (CPX) measurements

Subjects will undergo a maximum symptom-limited CPX, using cycle-ergometer ramp protocol (Corival, Lode, Netherlands) and ventilatory expired gas analysis cart (Quark CPET, Cosmed, Italy) following the recommendations of the European Respiratory Society for chronic lung diseases to obtain exercise capacity variables and respiratory efficiency. Volume and gas calibration will 
be performed before each test. Minute ventilation (VE), oxygen uptake $\left(\mathrm{VO}_{2}\right)$ and carbon dioxide output $\left(\mathrm{VCO}_{2}\right)$ will be acquired breath-by-breath and averaged over $10 \mathrm{~s}$ intervals. The ventilatory anaerobic threshold will be determined using the V-slope method. Peak $\mathrm{VO}_{2}$ will be expressed as the highest $10 \mathrm{~s}$ averaged sample obtained during the final plateau if the patient reached it, or the last $20 \mathrm{~s}$ of testing if not. The ventilatory efficiency (VE/ $\mathrm{VCO}_{2}$ slope) will be calculated from a linear regression equation, from the start of the test to the exercise peak. The ventilatory reserve will be determined from the forced expiratory volume in one second $\left(\mathrm{FEV}_{1}\right)$ (calculated as $\left.100-\left[\operatorname{VE}\left(\mathrm{FEV}_{1} \times 40\right) 100\right]\right)$. Circulatory power will be calculated from the $\mathrm{VO}_{2}$ and systolic blood pressure product at the peak, and ventilatory power from the quotient of peak systolic blood pressure and $\mathrm{VE} / \mathrm{VCO}_{2}$ slope. ${ }^{12}$

\section{Secondary outcomes}

\section{Dyspnoea}

Dyspnoea will be assessed using the modified Medical Research Council Dyspnoea Scale (mMRC). The mMRC is a $0-4$ scale used to classify dyspnoea's impact on physical function in patients with respiratory limitations. On mMRC, 0 represents a person who suffers from dyspnoea only with strenuous exercise, while 4 represents a person who is too breathless to leave the house or breathless when dressing or undressing. We chose the mMRC because of its easy application, and other studies with COVID-19 have used it to assess dyspnoea. ${ }^{47}$

\section{Respiratory muscle strength}

Respiratory muscle strength will be determined from residual volume (RV) using the technique proposed by Black and Hyatt to measure MIP and S-Index. Patients will be seated and motivated to perform a maximal voluntary exhalation effort at $\mathrm{RV}$, and then a verbal command will be given to perform a maximal inspiratory effort, according to ATS standards. ${ }^{13}$ The patients will be oriented to perform 10 manoeuvres, with $60 \mathrm{~s}$ rest intervals between the manoeuvres to avoid fatigue of the inspiratory muscles.

Inspiratory muscle endurance will be assessed using a timed inspiratory endurance test, which consists of breathing against a submaximal load ( $40 \%$ of MIP) provided by the device (KH2, POWERbreathe, UK) until task failure (Tlim in seconds).

\section{Anxiety and depression}

Anxiety and depression will be measured using the Hospital Anxiety and Depression Scale, a 14-item screening questionnaire from which an anxiety and depression subscale can be derived. Subscore values of $>5$ points will identify increased symptoms of anxiety or depression; a total score $>9$ will be considered indicative of psychological distress. ${ }^{14}$

Health-related quality of life

HRQoL will be assessed with the EQ-5D-3L instrument, which is recognised as a validated generic HRQoL measure consisting of five dimensions, each with five levels of response. Each answer combination will be converted into a health utility score. ${ }^{15}$

The EQ-5D-3Ltool has good test-retest reliability, is simple to use and gives a single preference-based index value for health status that can be used for costeffectiveness analysis.

\section{Fatigue}

Fatigue will be captured using the Fatigue Severity Scale, a nine-item questionnaire validated to evaluate disabling fatigue. Each item is rated on a 7-point scale ranging, from strongly disagree to strongly agree. A total score is derived from all nine questions; a higher score indicates a greater impact of fatigue on everyday activities. ${ }^{16}$

\section{Pulmonary function testing}

Spirometry will be performed using a spirometer (Microlab 3.500; CareFusion, Yorba Linda, California, USA). Three forced expiration manoeuvres will be performed for validity and reproducibility purposes according to the ATS/ERS criteria, with patients sitting, in a room with controlled temperature, ambient pressure and relative humidity. The following variables will be analysed: (1) forced vital capacity (FVC, L); (2) $\mathrm{FEV}_{1}$ (L); and (3) $\mathrm{FEV}_{1}: \mathrm{FVC}$ ratio (\%). The obtained values will be recorded and compared with the predicted values for a Brazilian population.

Incremental cost:utility ratio (quality-adjusted life years (QALYs)) A cost-utility analysis using the EQ-5D-3L responses will be performed to generate QALYs, which will generate an incremental cost per QALY. An economic evaluation will be undertaken to explore and determine the incremental costs and benefits of the IMT added to PR programme over a 6-month time horizon. This follow-up period aims to capture the direct effects of the interventions and offer insights into outcomes and costs accrued in the months after the intervention is completed. Direct medical and overhead costs and indirect patient costs will be estimated. Direct costs were estimated using time-and-motion analysis, and included procedure personnel and supplies.

An incremental analysis will be undertaken to calculate the difference in costs and the difference in outcomes (improvements in effectiveness measures and QALYs) associated with the IMT and PR programmes. QALYs will be calculated as the area under the curve connecting the utility scores reported at baseline and the subsequent follow-up points. ${ }^{17}$ The results will be presented in the form of incremental cost-effectiveness ratios, reflecting the extra cost for an additional unit of outcome. A sensitivity analysis will be performed to assess the robustness of the results to different values.

\section{Adverse events}

Adverse events are defined as any untoward medical occurrence in a patient or clinical investigation subject administered an investigational intervention, and any such event does not necessarily need to have a causal 


\begin{tabular}{|c|c|c|c|c|c|c|}
\hline \multirow[b]{3}{*}{ TIMEPOINT** } & \multicolumn{6}{|c|}{ STUDY PERIOD } \\
\hline & \multirow{2}{*}{$\begin{array}{c}\text { Enrolment } \\
-t_{1}\end{array}$} & \multirow{2}{*}{$\begin{array}{c}\text { Allocation } \\
0\end{array}$} & \multicolumn{4}{|c|}{ Post-allocation } \\
\hline & & & $\begin{array}{c}t_{1} \\
\text { Baseline }\end{array}$ & $\begin{array}{c}t_{2} \\
8 \\
\text { Weeks } \\
\end{array}$ & $\begin{array}{c}\text { t3 } \\
16 \text { weeks }\end{array}$ & $\begin{array}{c}t_{x} \\
6 \text { Months }\end{array}$ \\
\hline \multicolumn{7}{|l|}{ ENROLMENT: } \\
\hline \multirow{3}{*}{$\begin{array}{l}\text { Eligibility screen } \\
\text { Informed consent }\end{array}$} & $x$ & & & & & \\
\hline & $\mathrm{x}$ & & & & & \\
\hline & & $\mathrm{x}$ & & & & \\
\hline \multicolumn{7}{|l|}{ INTERVENTIONS: } \\
\hline \multicolumn{7}{|l|}{$\begin{array}{c}\text { [IMT + Pulmonary } \\
\text { Rehabilitation] }\end{array}$} \\
\hline \\
\hline \multicolumn{7}{|l|}{ ASSESSMENTS: } \\
\hline \multirow{2}{*}{$\begin{array}{r}\text { [Clinical and } \\
\text { Demographic } \\
\text { Characteristics] } \\
\text { [Cardiopulmonary } \\
\text { Exercise Testing] }\end{array}$} & $\mathrm{x}$ & & & & & \\
\hline & & & $x$ & $\mathrm{x}$ & $x$ & \\
\hline \multirow[t]{2}{*}{ [mMRC] } & & & $x$ & $\mathrm{x}$ & $\mathrm{x}$ & \\
\hline & & & $x$ & $x$ & $x$ & \\
\hline [EQ 5D 3L] & & & $x$ & $x$ & $x$ & \\
\hline HADS & & & $\mathrm{x}$ & $\mathrm{x}$ & $\mathrm{x}$ & \\
\hline $\begin{array}{r}F S S \\
\text { Inspiratory Muscle }\end{array}$ & & & $\mathrm{x}$ & $\mathrm{x}$ & $\mathrm{x}$ & \\
\hline \multirow{2}{*}{$\begin{array}{r}\text { Inspiratory Muscle } \\
\text { Endurance } \\
\text { Cost-utility } \\
\text { analysis }\end{array}$} & & & $x$ & $\mathrm{x}$ & $\mathrm{x}$ & \\
\hline & & & & & & $\mathrm{x}$ \\
\hline
\end{tabular}

Figure 2 Template of recommended content for the schedule of enrolment, interventions and assessments. FSS, Fatigue Severity Scale; HADS, Hospital Anxiety and Depression Scale; IMT, Inspiratory muscle training; mMRC, Medical Research Council Dyspnoea Scale.

relationship with this intervention. It is recognised that the patient population with a critical illness will experience several common signs and symptoms due to the severity of the underlying illness and the impact of standard therapies. These will not necessarily constitute an adverse event unless they are of concern or related to the study of the intervention in the investigator's clinical judgement. In all cases, the condition or disease underlying the symptoms, signs or laboratory values should be reported, for example, tachycardia rather than chest palpitations. Following each intervention and outcome measure session, trial staff will be required to complete data entry forms indicating a severe or minor adverse event. In the case of serious adverse events, the study chief investigator will be notified immediately; participants will be managed appropriately; and the incident will be reported to the relevant hospital ethics committee. Adverse events will be collected from randomisation to 48 hours after cessation of our study protocol through phone calls.

\section{Follow-up}

Outcomes will be assessed at baseline, 8 and 16 weeks, and 6 months postrandomisation. Figure 2 illustrates the schedule assessments of the participants. Patient-reported outcomes will also be collected during follow-up assessments. If any participant cannot attend the clinic, a postal questionnaire will be used to collect patient-reported outcomes.

\section{Sample size}

Due to the lack of data on the effect of supervised IMT in a PR in patients with post-COVID-19, we performed a power analysis based on a previous meta-analysis of peak $\mathrm{VO}_{2}$ (in $\mathrm{mL} / \mathrm{kg} / \mathrm{min}$ ) in patients with idiopathic pulmonary fibrosis. ${ }^{18}$ We used the software GPower to calculate sample size with a priori analysis with an intergroup difference of $1.46 \mathrm{~mL} / \mathrm{kg} / \mathrm{min}$, an $\mathrm{SD}$ of $3.5 \mathrm{~mL} / \mathrm{kg} /$ min, an $\alpha$ error probability of 0.05 and power (1- $\beta$ error probability) of 0.80 . Based on the results, it was decided that 138 patients will be divided into two groups, with a dropout rate of $15 \%$.

\section{Data analysis}

Data will be summarised and reported following the Consolidated Standards of Reporting Trials guidelines for RCTs, using intention-to-treat analyses. ${ }^{19}$ The Kolmogorov-Smirnov test will be used to analyse the normality of the sample's data. The patient baseline characteristics and outcome variables (both primary and secondary outcomes) will be summarised using descriptive measures of central tendency and dispersion for quantitative variables and absolute and relative frequencies for qualitative variables. Possible correlations will be assessed using Pearson or Spearman tests depending on the normality of the data. Associations will be performed using the $\chi^{2}$ or Fischer's exact test. Treatment effects or differences between the outcomes (at baseline and after 8 and 16weeks) of the study groups will be analysed using an ordinary two-way analysis of variance (ANOVA), if the sample is parametric. If the sample has a nonparametric distribution, paired t-tests and an ordinary one-way ANOVA will be used. Intergroup analyses will be performed using t-test or the Wilcoxon test, depending on the normality of the data.

\section{Trial managing and data monitoring}

The trial management group, consisting of project staff and coinvestigators involved in the day-to-day running of the trial, will meet monthly throughout the project to ensure data integrity, participant safety and evaluation of any adverse events.

Deidentified data and statistical code will be made available on request soon after each report of the data has been published. Different aspects of the data will be published separately, which will determine when those data are made publicly available. A data-sharing agreement will require a commitment to using the data only for specified research purposes, securing the data appropriately and destroying the data after a nominated period.

\section{DISCUSSION}

Patients with chronic respiratory symptoms commonly develop deconditioning and weakness of the respiratory 
muscles, which are related to dyspnoea and exercise intolerance in this population. ${ }^{20}$ IMT may reduce neural respiratory drive and subsequently improve abnormal breathing patterns, which may equalise the relationship between respiratory muscle demand and energy supply to the respiratory muscles. ${ }^{21}$ Langer et al demonstrated that an 8-week home-based IMT programme improved inspiratory muscle strength and dyspnoea, reducing diaphragm activation during maximal exercise, which may be associated with an important physiological response for the inspiratory muscles after IMT, which is compatible with the decrease in motor unit recruitment to generate a given force as a result of respiratory muscle hypertrophy. ${ }^{10}$

While we acknowledge the value of traditional IMT protocols that use mechanical loading devices, we believe that the IMT training with biofeedback provided by the POWERbreathe KH2 device has the potential to provide additional clinical benefits because it can modulate all aspects of muscular performance, including strength, power and work capacity. The real-time biofeedback likely encourages the generation of higher pressures throughout a full inspiration, a feature that differs from other IMT methods. Thus, IMT via the POWERbreathe KH2 device can facilitate a more controlled breathing pattern with an improved gas exchange during and after training.

Anastasio et al investigated the mid-term impact of COVID-19 on respiratory function and functional capacity 4 months after infection and found respiratory muscle weakness, as reflected by the mean $\%$ predicted of MIP assessed in these patients (58\%). ${ }^{4}$ Moreover, both airway occlusion pressure $(\mathrm{P} 0.1)$ and $\mathrm{P}$ 0.1:MIP ratio were significantly lower in patients with COVID-19, indicating a possible neural drive impairment. A previous meta-analysis showed additional effects of IMT on clinically relevant outcomes in a subgroup of patients with respiratory muscle weakness. ${ }^{22}$ Another compelling fact is that addition of IMT could improve the attenuation of respiratory muscle metaboloreflex in these patients. The fatiguing contraction pattern could decrease locomotor muscle perfusion, with blood flow redistribution in favour of the respiratory muscles. ${ }^{23}$ This impairment may contribute to early peripheral muscle fatigue and lower exercise capacity. Therefore, strategies such as IMT that improve the capacity and dynamic function of the respiratory muscles should be effective in reducing dyspnoea and might also improve the exercise capacity in patients with chronic respiratory diseases. ${ }^{21}$ Therefore, we believe that the use of IMT added to PR programmes could improve exercise capacity in patients with post-COVID-19.

The results of our study will provide valuable information for clinical practice. First, it will provide clinicians and rehabilitation practitioners information regarding the effects of exercise rehabilitation on chronic respiratory symptoms. Second, it will help healthcare providers perform a cost-effectiveness analysis of the PR and IMT methods in patients with COVID-19; and third, patients with all viral types of pneumonia will be educated beforehand about the potential benefits or harmful effects of engaging in physical activity and exercise programmes that include IMT, so that they can make an informed decision.

\section{Author affiliations}

${ }^{1}$ Physical Therapy Department, UniEVANGELICA University, Anapolis, Brazil ${ }^{2}$ Human Movement and Rehabilitation Program, UniEVANGELICA University, Anapolis, Brazil

${ }^{3}$ Health Sciences Program, Escola Superior de Ciências da Saúde, Brasilia, Brazil ${ }^{4}$ Rehabilitation Sciences and Health and Technologies in Health Sciences Program, University of Brasilia, Brasilia, Brazil

${ }^{5}$ Physical Therapy, University of Miami School of Medicine, Miami, Florida, USA

${ }^{6}$ Hospital Regional da Asa Norte, Brasilia, Brazil

${ }^{7}$ Physical Education, UniEVANGELICA University, Anapolis, Brazil

${ }^{8}$ Health Sciences and Technologies, University of Brasilia, Brasilia, Brazil

Acknowledgements We thank the many research nurses and allied health professionals who will participate in patient recruitment, data collection and data entry.

Contributors VM, LC and GC: conceived and designed the experiment. JC, ACGBL and DBS: investigation and resources. ANdCS and GFBC: original draft preparation. MCO, MdMCC, MEML and RB: pulmonary rehabilitation programme. IOS and LVFO: original draft preparation.

Funding This work will be supported by Conselho Nacional de Desenvolvimento Científico e Tecnológico (CNPQ)/JBS grant number 09/2019.

Competing interests None declared.

Patient and public involvement Patients and/or the public were involved in the design, conduct, reporting or dissemination plans of this research. Refer to the Methods and analysis section for further details.

Patient consent for publication Not required.

Provenance and peer review Not commissioned; externally peer reviewed.

Open access This is an open access article distributed in accordance with the Creative Commons Attribution Non Commercial (CC BY-NC 4.0) license, which permits others to distribute, remix, adapt, build upon this work non-commercially, and license their derivative works on different terms, provided the original work is properly cited, appropriate credit is given, any changes made indicated, and the use is non-commercial. See: http://creativecommons.org/licenses/by-nc/4.0/.

\section{ORCID iDs}

Vinicius Maldaner http://orcid.org/0000-0002-7804-7517

Miriã Cândida Oliveira http://orcid.org/0000-0002-5302-1388

\section{REFERENCES}

1 Carfi A, Bernabei R, Landi F, et al. Persistent symptoms in patients after acute COVID-19. JAMA 2020;324:603-5.

2 Vitacca M, Lazzeri M, Guffanti E, et al. Italian suggestions for pulmonary rehabilitation in COVID-19 patients recovering from acute respiratory failure: results of a Delphi process. Monaldi Arch Chest Dis 2020;90. doi:10.4081/monaldi.2020.1444. [Epub ahead of print: 23 Jun 2020].

3 Miyazato Y, Morioka S, Tsuzuki S, et al. Prolonged and late-onset symptoms of coronavirus disease 2019. Open Forum Infect Dis 2020;7:ofaa507.

4 Anastasio F, Barbuto S, Scarnecchia E, et al. Medium-Term impact of COVID-19 on pulmonary function, functional capacity and quality of life. Eur Respir J 2021. doi:10.1183/13993003.04015-2020. [Epub ahead of print: 11 Feb 2021].

5 Polastri M, Nava S, Clini E, et al. COVID-19 and pulmonary rehabilitation: preparing for phase three. Eur Respir J 2020;55. doi:10.1183/13993003.01822-2020. [Epub ahead of print: 2506 2020].

6 Spruit MA, Holland AE, Singh SJ, et al. COVID-19: interim guidance on rehabilitation in the hospital and Post-Hospital phase from a European respiratory Society and American thoracic Society-coordinated international Task force. Eur Respir J 2020. 
doi:10.1183/13993003.02197-2020. [Epub ahead of print: 13 Aug 2020].

7 Pancera S, Galeri S, Porta R, et al. Feasibility and efficacy of the pulmonary rehabilitation program in a rehabilitation center: case report of a young patient developing severe COVID-19 acute respiratory distress syndrome. $J$ Cardiopulm Rehabil Prev 2020;40:205-8

8 Hanada M, Kasawara KT, Mathur S, et al. Aerobic and breathing exercises improve dyspnea, exercise capacity and quality of life in idiopathic pulmonary fibrosis patients: systematic review and metaanalysis. J Thorac Dis 2020;12:1041-55.

9 Spruit MA, Singh SJ, Garvey C, et al. An official American thoracic Society/European respiratory Society statement: key concepts and advances in pulmonary rehabilitation. Am J Respir Crit Care Med 2013;188:e13-64.

10 Langer D, Ciavaglia C, Faisal A, et al. Inspiratory muscle training reduces diaphragm activation and dyspnea during exercise in COPD. J Appl Physiol 2018;125:381-92.

11 Charususin N, Gosselink R, Decramer M, et al. Randomised controlled trial of adjunctive inspiratory muscle training for patients with COPD. Thorax 2018;73:942-50.

12 Radtke T, Crook S, Kaltsakas G, et al. ERS statement on standardisation of cardiopulmonary exercise testing in chronic lung diseases. Eur Respir Rev 2019;28. doi:10.1183/16000617.01012018. [Epub ahead of print: 31 Dec 2019].

13 Laveneziana P, Albuquerque A, Aliverti A, et al. ERS statement on respiratory muscle testing at rest and during exercise. Eur Respir $J$ 2019;53. doi:10.1183/13993003.01214-2018. [Epub ahead of print: 1306 2019].

14 Bjelland I, Dahl AA, Haug TT, et al. The validity of the hospital anxiety and depression scale. An updated literature review. J Psychosom Res 2002;52:69-77.
15 Buchholz I, Thielker K, Feng Y-S, et al. Measuring changes in health over time using the EQ-5D 3L and 5L: a head-to-head comparison of measurement properties and sensitivity to change in a German inpatient rehabilitation sample. Qual Life Res 2015;24:829-35.

16 Valderramas S, Camelier AA, Silva SAda, et al. Reliability of the Brazilian Portuguese version of the fatigue severity scale and its correlation with pulmonary function, dyspnea, and functional capacity in patients with COPD. J Bras Pneumol 2013;39:427-33.

17 Billingham LJ, Abrams KR, Jones DR. Methods for the analysis of quality-of-life and survival data in health technology assessment. Health Technol Assess 1999;3:1-152.

18 Dowman L, Hill CJ, Holland AE. Pulmonary rehabilitation for interstitial lung disease. Cochrane Database Syst Rev 2014;10:CD006322.

19 Murphy JFA. Consort 2010 statement on randomised controlled trials. Ir Med J 2010;103:132.

20 Hoffman M, Augusto VM, Eduardo DS, et al. Inspiratory muscle training reduces dyspnea during activities of daily living and improves inspiratory muscle function and quality of life in patients with advanced lung disease. Physiother Theory Pract 2019:1-11.

21 Charususin N, Dacha S, Gosselink R, et al. Respiratory muscle function and exercise limitation in patients with chronic obstructive pulmonary disease: a review. Expert Rev Respir Med 2018;12:67-79.

22 Azambuja AdeCM, de Oliveira LZ, Sbruzzi G. Inspiratory muscle training in patients with heart failure: what is new? systematic review and meta-analysis. Phys Ther 2020;100:2099-109.

23 Sheel AW, Derchak PA, Morgan BJ, et al. Fatiguing inspiratory muscle work causes reflex reduction in resting leg blood flow in humans. J Physiol 2001;537:277-89. 